ESAIM: PROCEEDINGS, September 2007, Vol.21, 98-107

Gabriel Caloz \& Monique Dauge, Editors

\title{
MAXIMUM-NORM STABILITY, SMOOTHING AND RESOLVENT ESTIMATES FOR PARABOLIC FINITE ELEMENT EQUATIONS
}

\author{
VIDAR THOMÉE ${ }^{1}$
}

Dedicated to Michel Crouzeix

\begin{abstract}
We survey work on stability and smoothing estimates in maximum-norm for spatially semidiscrete finite element approximations of a model parabolic equation, and related such estimates for the resolvent of the corresponding discrete elliptic operator. We end with a short discussion of stability of fully discrete time stepping methods.

Résumé. Nous présentons un bilan des résultats sur la stabilité et les effets régularisants, vus en norme du maximum, lors de la semi-discrétisation éléments finis d'un problème parabolique modèle. Nous montrons le lien avec les estimations de résolvante pour l'opérateur elliptique approché correspondant. Nous concluons par une considération rapide de la discrétisation totale.
\end{abstract}

\section{INTRODUCTION}

Our purpose in this paper is to survey the development of stability and smoothing estimates in maximumnorm of spatially semidiscrete finite element approximations of a parabolic equation. In doing so we shall emphasize the application of the theory of analytic semigroups in Banach space to relate such estimates to maximum-norm estimates for the resolvent of the corresponding discrete elliptic operator. We also give an example of the use of such resolvent estimates in showing maximum-norm stability of fully discrete time stepping methods. For simplicity and concreteness we restrict the presentation to the model heat equation in a convex domain with smooth boundary in the plane, and to piecewise linear finite elements, even though several of the results described are valid in greater generality; we refer to the papers quoted for details.

We consider thus the initial-boundary value problem for the homogeneous heat equation,

$$
u_{t}-\Delta u=0 \quad \text { in } \Omega, \quad \text { and } u=0 \quad \text { on } \partial \Omega, \quad \text { for } t>0, \quad \text { with } u(\cdot, 0)=v \quad \text { in } \Omega,
$$

where $\Omega$ is a convex domain in $\mathbb{R}^{2}$ with smooth boundary $\partial \Omega$. We shall often write this problem in variational form,

$$
\left(u_{t}, \varphi\right)+(\nabla u, \nabla \varphi)=0, \quad \forall \varphi \in H_{0}^{1}=H_{0}^{1}(\Omega), \quad t \geq 0, \quad \text { with } u(0)=v .
$$

\footnotetext{
${ }^{1}$ Department of mathematics, Chalmers University of Technology, S 41296 Göteborg, Sweden; e-mail: thomee@math.chalmers.se
}

(c) EDP Sciences, SMAI 2007 
We define the solution operator $E(t)$ of this problem by $u(t)=E(t) v$. We first recall some properties of this operator in $L_{2}$, and begin with its stability. More precisely, $E(t)$ is a contraction in $L_{2}$, or

$$
\|E(t) v\| \leq\|v\|, \quad \text { for } t \geq 0, \quad \text { where }\|v\|=\|v\|_{L_{2}}=\left(\int_{\Omega}|v(x)|^{2} d x\right)^{1 / 2} .
$$

To show this we apply the standard energy argument, i.e., we set $\varphi=u$ in (1.2) to obtain

$$
\frac{1}{2} \frac{d}{d t}\|u\|^{2}+\|\nabla u\|^{2}=0, \quad \text { for } t \geq 0
$$

and integrate to derive

$$
\|u(t)\|^{2}+2 \int_{0}^{t}\|\nabla u\|^{2} d s=\|v\|^{2}, \quad \text { for } t \geq 0
$$

which contains the desired result. The solution operator $E(t)$ also has the smoothing property

$$
\left\|E^{\prime}(t) v\right\|=\|\Delta E(t) v\| \leq C t^{-1}\|v\|, \quad \text { for } t>0
$$

To sketch the proof, we formally differentiate (1.2) with respect to $t$, choose $\varphi=u_{t}$, and multiply by $t^{2}$ to obtain

which yields

$$
\frac{1}{2} \frac{d}{d t}\left(t^{2}\left\|u_{t}\right\|^{2}\right)+t^{2}\left\|\nabla u_{t}\right\|^{2}=t\left\|u_{t}\right\|^{2}, \quad \text { for } t \geq 0
$$

$$
t^{2}\left\|u_{t}(t)\right\|^{2}+2 \int_{0}^{t} s^{2}\left\|\nabla u_{t}\right\|^{2} d s=2 \int_{0}^{t} s\left\|u_{t}\right\|^{2} d s=2 \int_{0}^{t} s\|\Delta u\|^{2} d s .
$$

Similarly, using (1.3) in the last step,

$$
t\|\nabla u(t)\|^{2}+2 \int_{0}^{t} s\|\Delta u\|^{2} d s=\int_{0}^{t}\|\nabla u\|^{2} d s \leq \frac{1}{2}\|v\|^{2} .
$$

Together these estimates show the desired result.

We shall discuss spatially semidiscrete, and briefly also fully discrete, approximations of (1.1) based on continuous, piecewise linear finite elements, defined on a family of regular triangulations $\mathcal{T}_{h}=\{\tau\}$ of $\bar{\Omega}$ into closed triangles $\tau$, with angles bounded below; the family $\mathcal{T}_{h}$ will often be assumed to be quasiuniform in the sequel. We set $h=\max _{\tau \in \mathcal{T}_{h}} h_{\tau}$, where $h_{\tau}=\operatorname{diam}(\tau)$, and assume that $\Omega_{h}=\operatorname{Int}\left(\bigcup_{\tau \in \mathcal{T}_{h}} \tau\right)$ has its boundary vertices on $\partial \Omega$. Quasiuniformity of the family $\left\{\mathcal{T}_{h}\right\}$ then means that there is a positive $c$ such that any $\tau \in \mathcal{T}_{h}$ contains a disk or radius $c h$. We associate with $\mathcal{T}_{h}$ the finite dimensional space

$$
S_{h}=\left\{\chi \in \mathcal{C}(\bar{\Omega}):\left.\chi\right|_{\tau} \text { linear, for } \tau \in \mathcal{T}_{h}, \chi=0 \text { on } \partial \Omega\right\}
$$

The semidiscrete finite element problem associated with (1.1) is then to find $u_{h}(t) \in S_{h}$ for $t \geq 0$ such that

$$
\left(u_{h, t}, \chi\right)+\left(\nabla u_{h}, \nabla \chi\right)=0, \quad \text { for } \chi \in S_{h}, t>0, \quad \text { with } u_{h}(\cdot, 0)=v_{h}, \quad \text { where }(v, w)=\int_{\Omega} v(x) \bar{w}(x) d x
$$

Introducing the negative definite discrete Laplacian $\Delta_{h}: S_{h} \rightarrow S_{h}$, by

$$
-\left(\Delta_{h} \psi, \chi\right)=(\nabla \psi, \nabla \chi), \quad \forall \psi, \chi \in S_{h},
$$

one may write the semidiscrete problem (1.4) as

$$
u_{h, t}-\Delta_{h} u_{h}=0, \quad \text { for } t>0, \quad \text { with } u_{h}(0)=v_{h} .
$$


In the same way as for (1.1) we define the solution operator $E_{h}(t)$ of $(1.4)$, or (1.6), by $u_{h}(t)=E_{h}(t) v_{h}$. By the same argument as in the continuous case, we have the stability and smoothing properties in $L_{2}$, namely

$$
\left\|E_{h}(t) v_{h}\right\|+t\left\|E_{h}^{\prime}(t) v_{h}\right\| \leq C\left\|v_{h}\right\|, \quad \text { for } t \geq 0
$$

Such properties are useful in deriving smooth and nonsmooth data error estimates in $L_{2}$ for this problem, e.g., with $v_{h}$ suitable,

$$
\left\|u_{h}(t)-u(t)\right\| \leq\left\{\begin{array}{l}
C h^{2}\|v\|_{H^{2}}, \quad \text { if } v=0 \quad \text { on } \partial \Omega \\
C h^{2} t^{-1}\|v\| .
\end{array}\right.
$$

The proof of these results also depend on the elliptic error estimate $\left\|R_{h} v-v\right\| \leq C h^{2}\|v\|_{H^{2}}$, where the Ritz projection $R_{h}: H_{0}^{1} \rightarrow S_{h}$ is defined by $\left(\nabla R_{h} v, \nabla \chi\right)=(\nabla v, \nabla \chi), \forall \chi \in S_{h}$. We shall not further discuss error estimates below.

This survey paper is devoted to maximum-norm estimates analogous to (1.7) for the solution of the semidiscrete problem (1.4), and to the relation between such estimates and estimates in the complex plane for the resolvent of the operator $-\Delta_{h}$. As a preparation we consider first the case of the continuous problem (1.1). It follows immediately by the maximum-principle that the solution operator $E(t)$ of $(1.1)$ is a contraction in the Banach space $\mathcal{C}_{0}(\bar{\Omega})=\{v \in \mathcal{C}(\bar{\Omega}): v=0$ on $\partial \Omega\}$, so that

$$
\|E(t) v\|_{\mathcal{C}} \leq\|v\|_{\mathcal{C}}=\sup _{x \in \Omega}|v(x)|, \quad \text { for } t \geq 0, \quad v \in \mathcal{C}_{0}(\bar{\Omega})
$$

It is a special case of a result of Stewart [18] that $E(t)$ is an analytic semigroup on $\mathcal{C}_{0}(\bar{\Omega})$. This follows from the resolvent estimate

$$
\left\|(\lambda I+\Delta)^{-1} v\right\|_{\mathcal{C}} \leq \frac{C}{1+|\lambda|}\|v\|_{\mathcal{C}}, \quad \text { for } \lambda \notin \Sigma_{\delta}=\{\lambda:|\arg \lambda| \leq \delta\}
$$

where $\delta \in\left(0, \frac{1}{2} \pi\right)$ is arbitrary. By semigroup theory (cf. Theorem 3.1 below) this implies the smoothing estimate

$$
\left\|E^{\prime}(t) v\right\|_{\mathcal{C}} \leq C t^{-1}\|v\|_{\mathcal{C}}, \quad \text { for } t>0, \quad v \in \mathcal{C}_{0}(\bar{\Omega})
$$

For an alternative approach to showing the analyticity of $E(t)$, and hence (1.9), see Ouhabaz [12].

The rest of the paper is organized as follows. In Section 2 we use a weighted norm energy technique to derive a basic maximum-norm stability estimate for (1.4) for quasiuniform triangulations, and state a corresponding smoothing estimate. In Section 3 we use the theory of analytic semigroups to relate such stability and smoothing estimates to resolvent estimates, and present more recent work for (1.4) depending on this approach. In Section 4 we discuss briefly results valid for nonquasiuniform triangulations, and finally, in Section 5 , we give an example of application of a resolvent estimate in the analysis of a fully discrete scheme.

\section{A BASIC MAXIMUM-NORM STABILITy ESTIMATE}

We now consider basic stability and smoothing properties with respect to the maximum-norm of the solution operator $E_{h}(t)$ of the semidiscrete problem (1.6). We first note that a maximum-principle, or a contraction estimate analogous to (1.8),

$$
\left\|E_{h}(t) v_{h}\right\|_{\mathcal{C}} \leq\left\|v_{h}\right\|_{\mathcal{C}}, \quad \text { for } t \geq 0, \quad v_{h} \in S_{h}
$$

cannot hold. This was shown by a counterexample in Thomée [19], and in a more precise form in Thomée and Wahlbin [21]. We shall briefly give the argument of the latter paper. We define a strictly interior node of $\mathcal{T}_{h}$ as one, all neighbors of which are interior nodes of $\mathcal{T}_{h}$. 
Theorem 2.1. Assume that the triangulation $\mathcal{T}_{h}$ has the property that each interior node has a neighbor that is strictly interior. Then the inequality (2.1) cannot hold for the solution operator $E_{h}(t)$ of the semidiscrete problem (1.4).

Proof. With $\left\{P_{j}\right\}_{j=1}^{N_{h}}$ the interior nodes of $\mathcal{T}_{h}$, and $\left\{\Phi_{j}\right\}_{j=1}^{N_{h}} \subset S_{h}$ the corresponding standard pyramid basis functions, we introduce the mass matrix $\mathcal{M}=\left(m_{i j}\right)$, with $m_{i j}=\left(\Phi_{i}, \Phi_{j}\right)$, and the stiffness matrix $\mathcal{S}=\left(s_{i j}\right)$, with $s_{i j}=\left(\nabla \Phi_{i}, \nabla \Phi_{j}\right)$. Writing $u_{h}(t)=\sum_{j=1}^{N_{h}} \alpha_{j}(t) \Phi_{j}$ for the solution with initial interior nodal values 1 , i.e., with $v_{h}=\sum_{j=1}^{N_{h}} \Phi_{j}$, we have from (1.4) that, for $\alpha(t)=\left(\alpha_{1}(t), \ldots, \alpha_{N_{h}}(t)\right)^{T}$,

$$
\mathcal{M} \alpha^{\prime}+\mathcal{S} \alpha=0, \quad \text { for } t \geq 0, \quad \text { with } \alpha(0)=\underline{1}=(1, \ldots, 1)^{T} .
$$

If (2.1) were to hold, then for $t=0$ we would have $\beta=\alpha^{\prime}(0) \leq 0$, elementwise, and we shall see that this is not possible. For this purpose we note that the row sums of $\mathcal{S}$ corresponding to a strictly interior node $P_{i}$ vanishes, since $\sum_{j=1}^{n} s_{i j}=\left(\nabla \Phi_{i}, \nabla \sum_{j=1}^{n} \Phi_{j}\right)=\left(\nabla \Phi_{i}, \nabla v_{h}\right)=0$, as $v_{h}=1$ on $\operatorname{supp}\left(\Phi_{i}\right)$. Hence, taking $t=0$ in $(2.2)$, we see that $\sum_{j=1}^{n} m_{i j} \beta_{j}=-\sum_{j=1}^{n} s_{i j}=0$. Here $m_{i j} \geq 0$, with $m_{i j}>0$ if and only if $j=i$ or if $P_{j}$ is a neighbor of $P_{i}$, so that $P_{i} P_{j}$ is an interior edge of $\mathcal{T}_{h}$, and thus the corresponding elements $\beta_{j}$ of the nonpositive vector $\beta$ have to vanish. Therefore, by our assumption on $\mathcal{T}_{h}$, we have $\beta \equiv 0$. But $\beta$ cannot vanish, since, from (2.2) with $t=0$, we would then have $\mathcal{S} \underline{1}=0$, whereas $\mathcal{S} \underline{1} \cdot \underline{1}=\left(\nabla v_{h}, \nabla v_{h}\right)>0$.

One may still ask if a stability estimate of the form (2.1), but with a constant factor $C>1$ in front of the norm on the right, may be valid for the discrete solution operator $E_{h}(t)$. A first attempt to show this was made in Schatz, Thomée and Wahlbin [16], where such an estimate, with a logarithmic factor in the bound, was derived, for quasiuniform triangulations. This was done by a weighted norm energy technique, and since this has been a model for our later work, we sketch the proof.

Theorem 2.2. Assume that the family of triangulations $\mathcal{T}_{h}$ is quasiuniform. Then, for the solution operator $E_{h}(t)$ of (1.4), we have

$$
\left\|E_{h}(t) v_{h}\right\|_{\mathcal{C}} \leq C \ell_{h}\left\|v_{h}\right\|_{\mathcal{C}}, \quad \text { for } t \geq 0, \quad \text { with } \ell_{h}=\max (1, \log (1 / h))
$$

Sketch of proof: We want to demonstrate that $\left|\left(E_{h}(t) v_{h}\right)(x)\right| \leq C \ell_{h}\left\|v_{h}\right\|_{\mathcal{C}}$ for all $x \in \Omega$. For this purpose we introduce a discrete delta-function $\delta_{h}^{x} \in S_{h}$, defined by $\left(\delta_{h}^{x}, \chi\right)=\chi(x), \forall \chi \in S_{h}$, and also a discrete fundamental solution, by $\Gamma_{h}^{x}(t)=E_{h}(t) \delta_{h}^{x} \in S_{h}$. The latter function satisfies $\left(E_{h}(t) v_{h}\right)(x)=\left(\Gamma_{h}^{x}(t), v_{h}\right)$, and hence

$$
\left|\left(E_{h}(t) v_{h}\right)(x)\right| \leq\left\|\Gamma_{h}^{x}(t)\right\|_{L_{1}}\left\|v_{h}\right\|_{\mathcal{C}}
$$

For the proof of the theorem it thus suffices to show

$$
\left\|\Gamma_{h}^{x}(t)\right\|_{L_{1}} \leq C \ell_{h}, \quad \text { for } t \geq 0 .
$$

This will be done by an energy argument, using the modified distance function $\rho_{h}^{x}(y)=\left(|x-y|^{2}+h^{2}\right)^{1 / 2}$ as a weight. Writing for brevity $\Gamma=\Gamma_{h}^{x}(t), \rho=\rho_{h}^{x}$, we have

$$
\|\Gamma(t)\|_{L_{1}} \leq\left\|\rho^{-1}\right\| \cdot\|\rho \Gamma(t)\| \leq C \ell_{h}^{1 / 2}\|\rho \Gamma(t)\|, \quad \text { where }\|\cdot\|=\|\cdot\|_{L_{2}}
$$

so it now suffices to demonstrate that

$$
\|\rho \Gamma(t)\| \leq C \ell_{h}^{1 / 2}, \quad \text { for } t \geq 0
$$

By our definitions we have

$$
\left(\Gamma_{t}, \chi\right)+(\nabla \Gamma, \nabla \chi)=0, \quad \forall \chi \in S_{h}, \quad \text { with } \Gamma(0)=\delta_{h}^{x} .
$$


Simple calculations show that

$$
\begin{aligned}
\frac{1}{2} \frac{d}{d t}\|\rho \Gamma\|^{2}+\|\rho \nabla \Gamma\|^{2} & =\left(\Gamma_{t}, \rho^{2} \Gamma\right)+\left(\nabla \Gamma, \nabla\left(\rho^{2} \Gamma\right)\right)-2(\nabla \Gamma, \rho \nabla \rho \Gamma) \\
& =\left(\Gamma_{t}, \rho^{2} \Gamma-\chi\right)+\left(\nabla \Gamma, \nabla\left(\rho^{2} \Gamma-\chi\right)\right)-2(\rho \nabla \Gamma, \nabla \rho \Gamma)=I_{1}+I_{2}+I_{3},
\end{aligned}
$$

where in the second step we have subtracted (2.4). We now choose $\chi=P_{h}\left(\rho^{2} \Gamma\right)$, where $P_{h}$ denotes the orthogonal $L_{2}$-projection onto $S_{h}$. Then $I_{1}=0$. For $I_{2}$ and $I_{3}$ one may use the inverse estimate $\|\nabla \chi\| \leq C h^{-1}\|\chi\|$ and the superapproximation bound $\left\|\nabla\left(\rho^{2} \chi-P_{h}\left(\rho^{2} \chi\right)\right)\right\| \leq C h(\|\chi\|+\|\rho \nabla \chi\|)$, valid for $\chi \in S_{h}$, and also $|\nabla \rho| \leq C$, to obtain

Combining this with (2.5) we find

$$
\left|I_{1}\right|+\left|I_{2}\right| \leq C\left(\|\Gamma\|^{2}+\|\Gamma\|\|\rho \nabla \Gamma\|\right) \leq \frac{1}{2}\|\rho \nabla \Gamma\|^{2}+C\|\Gamma\|^{2} .
$$

and hence by integration,

$$
\frac{d}{d t}\|\rho \Gamma\|^{2}+\|\rho \nabla \Gamma\|^{2} \leq C\|\Gamma\|^{2}
$$

$$
\|\rho \Gamma(t)\|^{2}+\int_{0}^{t}\|\rho \nabla \Gamma\|^{2} d s \leq\left\|\rho \delta_{h}^{x}\right\|^{2}+C \int_{0}^{t}\|\Gamma\|^{2} d s .
$$

Here, by simple energy arguments, after multiplication of (1.6) by $\left(-\Delta_{h}\right)^{-1}$, one may show that

$$
\int_{0}^{t}\|\Gamma\|^{2} d s \leq\left(\left(-\Delta_{h}\right)^{-1} \delta_{h}^{x}, \delta_{h}^{x}\right)=G_{h}^{x}(x), \quad \text { where } G_{h}^{x}=\left(-\Delta_{h}\right)^{-1} \delta_{h}^{x} .
$$

The function $G_{h}^{x} \in S_{h}$ may be thought of as a discrete Green's function, since

$$
\left(\nabla G_{h}^{x}, \nabla \chi\right)=-\left(\Delta_{h} G_{h}^{x}, \chi\right)=\left(\delta_{h}^{x}, \chi\right)=\chi(x), \quad \forall \chi \in S_{h} .
$$

Hence, using the "almost Sobolev inequality" $\|\chi\|_{\mathcal{C}} \leq C \ell_{h}^{1 / 2}\|\nabla \chi\|$, for $\chi \in S_{h}$, we obtain

$$
G_{h}^{x}(x) \leq C \ell_{h}^{1 / 2}\left\|\nabla G_{h}^{x}\right\|=C \ell_{h}^{1 / 2}\left(G_{h}^{x}(x)\right)^{1 / 2},
$$

so that $G_{h}^{x}(x) \leq C \ell_{h}$, which yields the desired bound for the integral in the right hand side of (2.6).

To complete the proof we shall show $\left\|\rho \delta_{h}^{x}\right\| \leq C$. For this we shall use the following exponential decay property of the $L_{2}$ projection onto $S_{h}$, which goes back to Descloux [9], and which was shown in this form in Crouzeix and Thomée [7]. Here, for $\tau_{0} \in \mathcal{T}_{h}$, we denote by $Q_{j}\left(\tau_{0}\right)$ the set of triangles which are " $j$ triangles away from $\tau_{0}$ ", defined by setting $Q_{0}\left(\tau_{0}\right)=\tau_{0}$ and then, recursively, for $j \geq 1, Q_{j}\left(\tau_{0}\right)$ to be the union of the closed triangles $\tau$ which are not in $\bigcup_{i<j} Q_{i}\left(\tau_{0}\right)$, but which have at least one vertex in $Q_{j-1}\left(\tau_{0}\right)$.

Lemma 2.1. Assume that $\operatorname{supp}(v) \subset \tau_{0} \in \mathcal{T}_{h}$. Then, with $\gamma=\sqrt{3}-\sqrt{2} \approx 0.318$, we have

$$
\left\|P_{h} v\right\|_{L_{2}(\tau)} \leq C \gamma^{j}\|v\|_{L_{2}}, \quad \text { for } \tau \in Q_{j}\left(\tau_{0}\right) .
$$

Now to bound $\rho \delta_{h}^{x}$, let $x \in \tau \subset Q_{j}\left(\tau_{0}\right)$. Then, for any $\varphi \in \mathcal{C}_{0}^{\infty}(\tau)$ with $\|\varphi\|=1$ we have, using Lemma 2.1,

$$
\left(\delta_{h}^{x}, \varphi\right)=\left(\delta_{h}^{x}, P_{h} \varphi\right)=\left(P_{h} \varphi\right)(x) \leq\left\|P_{h} \varphi\right\|_{\mathcal{C}(\tau)} \leq C h^{-1}\left\|P_{h} \varphi\right\|_{L_{2}(\tau)} \leq C h^{-1} \gamma^{j},
$$

and hence $\left\|\delta_{h}^{x}\right\|_{L_{2}(\tau)} \leq C h^{-1} \gamma^{j}$. For any $y \in \tau$ we also have $\rho(y) \leq C(j+1) h$ and hence, since the number of triangles in $Q_{j}\left(\tau_{0}\right)$ is bounded by $C(j+1)^{2}$,

$$
\left\|\rho \delta_{h}^{x}\right\|^{2} \leq \sum_{j \geq 0} \sup _{y \in Q_{j}\left(\tau_{0}\right)}\left(\rho(y)^{2}\left\|\delta_{h}^{x}\right\|_{Q_{j}\left(\tau_{0}\right)}^{2}\right) \leq C h^{2} \sum_{j \geq 0}(j+1)^{4} h^{-2} \gamma^{2 j} \leq C .
$$


Together these estimates show (2.3) and thus the theorem.

Using the same weighted norm technique as above one may also show the following smoothing property of the discrete solution operator.

Theorem 2.3. Assume that the family of triangulations $\mathcal{T}_{h}$ is quasiuniform. Then we have

$$
\left\|E_{h}^{\prime}(t) v_{h}\right\|_{\mathcal{C}} \leq C t^{-1} \ell_{h}\left\|v_{h}\right\|_{\mathcal{C}}, \quad \text { for } t>0
$$

In Thomée and Wahlbin [20] analogues of the results of Theorems 2.2 and 2.3 were shown in one space dimension, in which the logarithmic factor is $C \ell_{h}^{2}$. For other related stability results, see Nitsche and Wheeler [11], Rannacher [15], and Chen [4].

\section{Analytic Semigroups And Resolvent estimates}

We now want to put our stability problem into an abstract framework, and consider therefore an initial-value problem in a Banach space $\mathbb{B}$ with norm $\|\cdot\|$,

$$
u_{t}+A u=0, \quad \text { for } t>0, \quad \text { with } u(0)=v,
$$

where $A$ is a closed, densely defined linear operator in $\mathbb{B}$. This includes, e.g., the cases considered in the beginning of Section 1 , where $\mathbb{B}=L_{2}(\Omega), A=-\Delta$ and $\mathbb{B}=S_{h}, A=-\Delta_{h}$, with $\|\cdot\|=\|\cdot\|_{L_{2}}$, but since we want to apply this to discuss maximum-norm estimates, we will be particularly interested in the cases that $\mathbb{B}=\mathcal{C}_{0}(\bar{\Omega}), A=-\Delta$, and $\mathbb{B}=S_{h}, A=-\Delta_{h}$, with $\|\cdot\|=\|\cdot\|_{\mathcal{C}}$. We define the solution operator $E(t)$ of $(3.1)$ by $E(t) v=u(t)$, and assume that this is a bounded semigroup on $\mathbb{B}$, generated by $-A$, or $E(t)=e^{-A t}$.

We first quote a general theorem, see Pazy [14], Thomée [19], which shows that the smoothing property is equivalent to a resolvent estimate.

Theorem 3.1. Assume that $E(t)=e^{-A t}$ is a bounded semigroup on $\mathbb{B}$ which also has the smoothing property, so that

$$
\|E(t)\|+t\left\|E^{\prime}(t)\right\| \leq K, \quad \text { for } t \geq 0, \quad \text { with } K \geq 1
$$

Then there are $M \geq 1$ and $\delta \in\left(0, \frac{1}{2} \pi\right)$ such that the resolvent set $\rho(A)$ of $A$ containts $\mathbb{C} \backslash \Sigma_{\delta}$, and

$$
\left\|(\lambda I-A)^{-1}\right\| \leq \frac{M}{|\lambda|}, \quad \forall \lambda \notin \Sigma_{\delta} .
$$

One may choose $M=C K^{2}, \delta=\frac{1}{2} \pi-c K^{-2}$, with $C$, c positive constants independent of $K$.

Conversely, if the resolvent estimate (3.3) holds, with $M \geq 1, \delta \in\left(0, \frac{1}{2} \pi\right)$, then, with, e.g., $\Gamma=\partial \Sigma_{\psi}$, where $\psi \in\left(\delta, \frac{1}{2} \pi\right)$, the integral

$$
E(t)=\frac{1}{2 \pi i} \int_{\Gamma} e^{-\lambda t}(\lambda I-A)^{-1} d \lambda
$$

defines a bounded semigroup $E(t)=e^{-A t}$ in $\mathbb{B}$, which is the solution operator of (3.1), and, with $C$ a positive constant, independent of $M$ and $\delta$,

$$
\|E(t)\| \leq C M \max (1, \log (1 / \cos \delta)) \quad \text { and } t\left\|E^{\prime}(t)\right\| \leq C M / \cos \delta, \quad \text { for } t>0
$$

When (3.3) holds, $E(t)$ is, in fact, analytic as a function of $t$ in a sector around $\mathbb{R}_{+}$, and is therefore referred to as an analytic semigroup.

We shall now apply Theorem 3.1 to discuss maximum-norm stability and smoothness properties for the solution operator $E_{h}(t)$ of (1.4), and related estimates for the resolvent of the discrete Laplacian. We first note 
that by Theorems 2.2 and 2.3, and with $K=C \ell_{h}$, the first part of Theorem 3.1 implies a resolvent estimate for $-\Delta_{h}$, namely

$$
\left\|\left(\lambda I+\Delta_{h}\right)^{-1} v_{h}\right\|_{\mathcal{C}} \leq \frac{C \ell_{h}^{2}}{|\lambda|}\left\|v_{h}\right\|_{\mathcal{C}}, \quad \forall \lambda \notin \Sigma_{\delta_{h}}, \quad \text { where } \delta_{h}=\frac{1}{2} \pi-c \ell_{h}^{-2}
$$

On the other hand, if this estimate holds, then the second part of Theorem 3.1, with $M=C \ell_{h}^{2}, \delta_{h}=\frac{1}{2} \pi-c \ell_{h}^{-2}$, implies the stability and smoothing estimates

$$
\left\|E_{h}(t) v_{h}\right\|_{\mathcal{C}} \leq C \ell_{h}^{2} \max \left(1, \log \ell_{h}\right)\left\|v_{h}\right\|_{\mathcal{C}} \quad \text { and } \quad t\left\|E_{h}^{\prime}(t) v_{h}\right\|_{\mathcal{C}} \leq C \ell_{h}^{4}\left\|v_{h}\right\|_{\mathcal{C}}
$$

which, however, are weaker than those of Theorems 2.2 and 2.3 .

Using the same weighted norm technique as in the proofs of Theorems 2.2 and 2.3, one may also directly show he following resolvent estimate, see [19].

Theorem 3.2. Assume the family of triangulations $\mathcal{T}_{h}$ is quasiuniform. Then, with $\Delta_{h}$ defined in (1.5),

$$
\left\|\left(\lambda I+\Delta_{h}\right)^{-1} v_{h}\right\|_{\mathcal{C}} \leq \frac{C \ell_{h}}{1+|\lambda|}\left\|v_{h}\right\|_{\mathcal{C}}, \quad \forall \lambda \notin \Sigma_{\varphi}, \quad \forall \varphi \in\left(0, \frac{1}{2} \pi\right)
$$

By the second part of Theorem 3.1, this provides a new proof of Theorems 2.2 and 2.3. In Chatzipantelidis, Lazarov, Thomée and Wahlbin [3], it was shown that the resolvent estimate (3.5) holds also when $\Omega$ is an arbitrary plane polygonal domain, not necessarily convex.

In Thomée and Wahlbin [22] it was demonstrated that the logarithmic factor in Theorems 2.2 and 2.3 may be removed; this was done first in Schatz, Thomée and Wahlbin [17] in the technically somewhat simpler case of natural (Neumann) boundary conditions, and for a more general elliptic operator. We thus have the following.

Theorem 3.3. Assume that the $\mathcal{T}_{h}$ form a quasiuniform family. Then, for $E_{h}(t)=e^{\Delta_{h} t}$, we have, with $C$ independent of $h$,

$$
\left\|E_{h}(t) v_{h}\right\|_{\mathcal{C}}+t\left\|E_{h}^{\prime}(t) v_{h}\right\|_{\mathcal{C}} \leq C\left\|v_{h}\right\|_{\mathcal{C}}, \quad \text { for } t \geq 0
$$

The proof, which is quite technical, uses a norm with a weight function depending on both $x$ and $t$. In both [17] and [22] higher order elements were also covered.

By Theorem 3.1, (3.6) implies that there exists $\varphi \in\left(0, \frac{1}{2} \pi\right)$ such that

$$
\left\|\left(\lambda I+\Delta_{h}\right)^{-1} v_{h}\right\|_{\mathcal{C}} \leq \frac{C}{1+|\lambda|}\left\|v_{h}\right\|_{\mathcal{C}}, \quad \forall \lambda \notin \Sigma_{\varphi}
$$

On the other hand, by the second part of Theorem 3.1, (3.7) would provide an alternative proof of Theorem 3.3. In Bakaev, Thomée and Wahlbin [1] a direct proof was given that such an estimate holds outside an arbitrary sector containing the positive real axis:

Theorem 3.4. Assume that the $\mathcal{T}_{h}$ form a quasiuniform family. Then (3.7) holds for any $\varphi \in\left(0, \frac{1}{2} \pi\right)$.

The result in [1] was shown for arbitrary space dimension; in one dimension, the estimate was derived in Crouzeix, Larsson and Thomée [5] for a more general elliptic operator, and also for higher order elements. For an early result using a resolvent estimate, in one space dimension, see Wahlbin [23].

\section{RESUlts FOR NONQUASIUNIFORM TRIANGULATIONS.}

In all the results quoted above the family of triangulations $\mathcal{T}_{h}$ was required to be quasiuniform, which is a somewhat undesirable restriction. A first attempt to weaken this requirement was made in Crouzeix and 
Thomée [8], where a resolvent estimate of the desired type, with a logarithmic factor, was shown for a modified discrete Laplacian, defined by

$$
-\left(\Delta_{h} \psi, \chi\right)_{h}=(\nabla \psi, \nabla \chi), \quad \forall \psi, \chi \in S_{h},
$$

where $(\cdot, \cdot)_{h}$ denotes a simple quadrature approximation of the $L_{2}$-inner product,

$$
(\psi, \chi)_{h}=\sum_{\tau \in \mathcal{T}_{h}} Q_{\tau, h}(\psi \chi), \quad \text { where } Q_{\tau, h}(f)=\frac{1}{3} \sum_{j=1}^{3} f\left(P_{\tau, j}\right) \text { area }(\tau) \approx \int_{\tau} f d x
$$

and for triangulations of Delaunay type. Such triangulations $\mathcal{T}_{h}$ are characterized by the property that, for each pair of triangles with a common interior edge, the opposite angles $\alpha$ and $\beta$ satisfy $\alpha+\beta \leq \pi$, and families of such $\mathcal{T}_{h}$ do not need to be quasiuniform. This choice of the discrete Laplacian corresponds to the so-called lumped mass modification of the semidiscrete problem (1.4), defined by

$$
\left(u_{h, t}, \chi\right)_{h}+\left(\nabla u_{h}, \nabla \chi\right)=0, \quad \text { for } \chi \in S_{h}, t>0, \quad \text { with } u_{h}(\cdot, 0)=v_{h} \quad \text { in } \Omega .
$$

This modification may also be expressed by saying that the mass matrix $\mathcal{M}=\left(m_{i j}\right)$ is replaced by diagonal matrix $\overline{\mathcal{M}}=\left(\bar{m}_{i j}\right), \bar{m}_{i j}=\left(\Phi_{i}, \Phi_{j}\right)_{h}$, with diagonal elements $\bar{m}_{i i}=\sum_{j=1}^{N_{h}} m_{i j}$, and $\bar{m}_{i j}=0$ if $j \neq i$.

For this problem a maximum principle holds, and the solution operator $\bar{E}_{h}(t)=e^{\bar{\Delta}_{h} t}$ is a contraction with respect to the maximum-norm, which is a result that goes back to Fujii [10], cf. [19].

Theorem 4.1. Assume $\mathcal{T}_{h}$ is of Delaunay type. Then a maximum-principle holds for (4.2) and

$$
\left\|\bar{E}_{h}(t) v_{h}\right\|_{\mathcal{C}} \leq\left\|v_{h}\right\|_{\mathcal{C}}, \quad \text { for } t \geq 0
$$

It was shown in Crouzeix and Thomée [8] that this contraction semigroup is, in fact, an analytic semigroup.

Theorem 4.2. Assume $\mathcal{T}_{h}$ is of Delaunay type. Then, for $\bar{\Delta}_{h}$ defined by (4.1), and with $C$, c positive constants, independent of $h$, and $h_{\min }=\min _{\tau \in \mathcal{T}_{h}} h_{\tau}$, we have

$$
\left\|\left(\lambda I+\bar{\Delta}_{h}\right)^{-1} v_{h}\right\|_{\mathcal{C}} \leq \frac{C \ell_{h}^{1 / 2}}{1+|\lambda|}\left\|v_{h}\right\|_{\mathcal{C}}, \quad \lambda \notin \Sigma_{\delta_{h}}, \quad \delta_{h}=\frac{\pi}{2}-c \ell_{h}^{1 / 2}, \quad \text { where } \ell_{h}=\max \left(1, \log \left(1 / h_{\text {min }}\right)\right) .
$$

The proof uses energy arguments to show first the corresponding estimate in a discrete $L_{p}$ norm, for large $p<\infty$, and then applies a local inverse estimate. In one space dimension, the corresponding result was shown in [8], without a logarithmic factor.

It follows from the second part of Theorem 3.1 that $\bar{E}_{h}(t)$ has the smoothing property

$$
\left\|\bar{E}_{h}^{\prime}(t) v_{h}\right\|_{\mathcal{C}} \leq C \ell_{h} t^{-1}\left\|v_{h}\right\|_{\mathcal{C}}, \quad \text { for } t>0
$$

We now turn to results for the standard finite element method in the case of nonquasiuniform triangulations, and quote the following result of Bakaev, Crouzeix and Thomée [2].

Theorem 4.3. Let $Q_{j}\left(\tau_{0}\right)$ be as in Lemma 2.1, and let $n_{j}\left(\tau_{0}\right)$ be the number of triangles in $Q_{j}\left(\tau_{0}\right)$. Assume that $\mathcal{T}_{h}$ satisfies, for some $\alpha \geq 1, \beta \geq 1$,

$$
h_{\tau} / h_{\tau_{0}} \leq C \alpha^{j}, \quad \text { for } \tau \in Q_{j}\left(\tau_{0}\right), \quad \text { and } n_{j}\left(\tau_{0}\right) \leq C \beta^{j}, \quad \forall \tau_{0} \in \mathcal{T}_{h} .
$$

Then, if $\alpha^{2} \beta \gamma<1$, we have, with $\Delta_{h}$ defined in (1.5),

$$
\left\|\left(\lambda I+\Delta_{h}\right)^{-1} v_{h}\right\|_{\mathcal{C}} \leq \frac{C \ell_{h}^{1 / 2}}{1+|\lambda|}\left\|v_{h}\right\|_{\mathcal{C}}, \quad \forall \lambda \notin \Sigma_{\delta}, \quad \text { for any } \delta \in\left(0, \frac{1}{2} \pi\right) .
$$


We remark that the $\mathcal{T}_{h}$ are quasiuniform if $\alpha=1$, and that we may always choose $\beta=\alpha^{4}$ in $(4.3)$ if $\alpha>1$. With $\gamma=0.318$ as in Lemma 2.1, and $\beta=\alpha^{4}$, the condition $\alpha^{2} \beta \gamma<1$ holds if $\alpha<1.21$, which permits seriously nonquasiuniform families of $\mathcal{T}_{h}$. For one space dimension the corresponding result was shown in [2], without a logarithmic factor.

The proof uses again weighted norm energy arguments, and depends essentially on the exponential decay property of the $L_{2}$-projection $P_{h}$ in Lemma 2.1, which remains valid in the present situation. Lemma 2.1 was used in [7] to show the maximum-norm stability of $P_{h}$ under the assumption (4.3), with $\alpha \beta \gamma<1$.

\section{Time Stepping. Fully Discrete SCHEMeS}

In this closing section we shall see that resolvent estimates of the type quoted above may also be used in the analysis of fully discrete schemes for (1.1), obtained by time stepping in the spatially semidiscrete equation (1.4). It is convenient to treat the time stepping in our above Banach space framework, and we consider thus an initial value problem of the form (3.1) in a Banach space $\mathbb{B}$ with norm $\|\cdot\|$. We assume that $A$ is a closed, densely defined linear operator, such that its resolvent satisfies (3.3) for some $M \geq 1, \delta \in\left(0, \frac{1}{2} \pi\right)$. By Theorem 3.1, the operator $-A$ then generates an analytic semigroup $E(t)$ defined by $(3.4)$, where, e.g., $\Gamma=\partial \Sigma_{\psi}, \psi \in\left(\delta, \frac{1}{2} \pi\right)$, which is the solution operator of (3.1).

We shall now discuss discretization in time of (3.1), where $A$ satisfies (3.3). Letting $k$ denote a time step and $t_{n}=n k$, and letting $r(\lambda)$ be a rational function which is bounded on $\Sigma_{\psi}$ with $\psi \in\left(\delta, \frac{1}{2} \pi\right)$, we define our discrete time approximation $U_{k}^{n}$ of $u\left(t_{n}\right)=E\left(t_{n}\right) v=e^{-A t_{n}} v$ by the recursion formula

$$
U_{k}^{n+1}=E_{k} U_{k}^{n}, \quad \text { for } n \geq 0, \quad \text { where } E_{k}=r(k A), \quad \text { with } U_{k}^{0}=v .
$$

The time discrete approximate solution of (3.1) may thus be written as $U_{k}^{n}=E_{k}^{n} v$. We have the following result from Crouzeix, Larsson, Piskarev and Thomée [6], see also Palencia [13].

Theorem 5.1. Let $U_{k}^{n}$ be defined by (5.1), and assume that the resolvent estimate (3.3) holds for some $M \geq$ $1, \delta \in\left(0, \frac{1}{2} \pi\right)$, and that $r(\lambda)$ is $A(\theta)-$ stable, with $\theta \in\left(\delta, \frac{1}{2} \pi\right]$. Then

$$
\left\|U_{k}^{n}\right\|=\left\|E_{k}^{n} v\right\| \leq C M\|v\|, \quad \text { for } t_{n} \geq 0 .
$$

The proof uses the fact that, for any rational function $R(\lambda)$, bounded in $\Sigma_{\theta}$, one has, with $\Gamma$ suitable,

$$
R(A)=R(\infty) I+\frac{1}{2 \pi i} \int_{\Gamma} R(\lambda)(\lambda I-A)^{-1} d \lambda .
$$

This is then used with $R(\lambda)=r(k \lambda)^{n}$, and the resolvent estimate (3.3) is applied. Special consideration is needed with $\mid r(\infty \mid=1$.

As an example, for the Crank-Nicolson method for discretization in time of the spatially semidiscrete equation (1.6) in time, corresponding to using the $A$-stable rational function $r(\lambda)=\left(1+\frac{1}{2} \lambda\right)^{-1}\left(1-\frac{1}{2} \lambda\right)$, we obtain, for instance, under the assumptions of Theorem 4.3, that, for the fully discrete solution,

$$
\left\|U_{k h}^{n}\right\|_{\mathcal{C}} \leq C \ell_{h}^{1 / 2}\left\|v_{h}\right\|_{\mathcal{C}}, \quad \text { where } U_{k h}^{n}=E_{k h}^{n} v_{h}, \quad \text { with } E_{k h}=r\left(-k \Delta_{h}\right) .
$$

\section{REFERENCES}

[1] N.Yu. Bakaev, V. Thomée, and L.B. Wahlbin, Maximum-norm estimates for resolvents of elliptic finite element operators, Math. Comp. 72 (2002), 1597-1610.

[2] N.Yu. Bakaev, M. Crouzeix and V. Thomée, Maximum-norm resolvent estimates for elliptic finite element operators on nonquasiuniform triangulations, M2AN 40 (2006), 923-938.

[3] P. Chatzipantelidis, R.D. Lazarov, V. Thomée, and L.B. Wahlbin, Parabolic finite element equations in nonconvex polygonal domains, BIT, 6 (2006), 113-143. 
[4] H. Chen, An $L^{2}$ and $L^{\infty}$-Error Analysis for Parabolic Finite Element Equations with Application by Superconvergence and Error Expansions, Doctoral Dissertation, Heidelberg University, 1993.

[5] M. Crouzeix, S. Larsson and V. Thomée, Resolvent estimates for elliptic finite element operators in one dimension, Math. Comp. 63 (1994), 121-140.

[6] M. Crouzeix, S. Larsson, S. Piskarev, and V. Thomée, The stability of rational approximations of analytic semigroups, BIT 33 (1993), 74-84..

[7] M. Crouzeix and V. Thomée, The stability in $L_{p}$ and $W_{p}^{1}$ of the $L_{2}$-projection onto finite element function spaces, Math. Comp. 48 (1987), 521-532.

[8] M. Crouzeix and and V. Thomée, Resolvent estimates in $l_{p}$ for discrete Laplacians on irregular meshes and maximum-norm stability of parabolic finite difference schemes, CMAM 1 (2001), 3-17.

[9] J. Descloux, On finite element matrices, SIAM J. Numer. Anal., 9 (1972), 260-265.

[10] H. Fujii, Some remarks on finite element analysis of time-dependent field problems, in Theory and Practice in Finite Element Structural Analysis, University of Tokyo Press, Tokyo, 1973, pp. 91-106.

[11] J.A. Nitsche and M.F. Wheeler, $L_{\infty}$-boundedness of the finite element Galerkin operator for parabolic problems, Numer. Funct. Anal. Optim. $\underline{\mathrm{f}} 4$ (1981-82), 325-353.

[12] E.M. Ouhabaz, Analysis of Heat Equation on Domains, LMS Monograph Series 31, Princeton University Press, Princeton 2005.

[13] C. Palencia, A stability result for sectorial operators in Banach spaces, SIAM J. Numer. Anal. 30 (1993), 1373-1384.

[14] A. Pazy, Semigroups of Linear Operators and Applications to Partial Differential Equations, Springer-Verlag, New York, 1983.

[15] R. Rannacher, $L^{\infty}$-stability estimates and asymptotic error expansion for parabolic finite element equations, in Extrapolation and Defect Correction (1990), Bonner Math. Schriften No. 228, University of Bonn, 1991.

[16] A.H. Schatz, V. Thomée, and L.B. Wahlbin, Maximum norm stability and error estimates in parabolic finite element equations, Comm. Pure Appl. Math. 33 (1980), 265-304.

[17] A.H. Schatz, V. Thomée, and L.B. Wahlbin, Stability, analyticity, and almost best approximation in maximum-norm for parabolic finite element equations, Comm. Pure Appl. Math. 51 (1998), 1349-1385.

[18] H.B. Stewart, Generation of analytic semigroups by strongly elliptic operators, Trans. Amer. Math. Soc. 199 (1974), $141-161$.

[19] V. Thomée, Galerkin Finite Element Methods for Parabolic Problems, Second edition, Springer-Verlag, Berlin Heidelberg, 2006.

[20] V. Thomée and L.B. Wahlbin, Maximum norm stability and error estimates in Galerkin methods for parabolic equations in one space variable, Numer. Math. 41 (1983), 345-371.

[21] V. Thomée and L.B. Wahlbin, Stability and analyticity in maximum-norm for simplicial Lagrange finite element semidiscretizations of parabolic equations with Dirichlet boundary conditions, Numer. Math. 87 (2000), 373-389.

[22] V. Thomée and L.B. Wahlbin, On the existence of maximum principles in parabolic finite element equations, to appear in Math. Comp.

[23] L.B. Wahlbin, A quasioptimal estimate in piecewise polynomial Galerkin approximation of parabolic problems, in Numerical Analysis (Dundee, 1981), Lecture Notes in Mathematics No. 912, Springer-Verlag, Berlin-Heidelberg, 1982, pp. 230-245. 